\title{
Algebraic singularities of scattering amplitudes from tropical geometry
}

\author{
James Drummond, ${ }^{a}$ Jack Foster, ${ }^{a}$ Ömer Gürdoğan ${ }^{b}$ and Chrysostomos Kalousios ${ }^{a}$ \\ ${ }^{a}$ School of Physics $\&$ Astronomy, University of Southampton, \\ Highfield, Southampton, SO17 1BJ, U.K. \\ ${ }^{b}$ Mathematical Institute, University of Oxford, \\ Andrew Wiles Building, Woodstock Road, Oxford, OX2 6GG, U.K. \\ E-mail: j.m.drummond@soton.ac.uk, j.a.foster@soton.ac.uk, \\ omer.gurdogan@maths.ox.ac.uk, c.kalousios@soton.ac.uk
}

ABSTRACT: We address the appearance of algebraic singularities in the symbol alphabet of scattering amplitudes in the context of planar $\mathcal{N}=4$ super Yang-Mills theory. We argue that connections between cluster algebras and tropical geometry provide a natural language for postulating a finite alphabet for scattering amplitudes beyond six and seven points where the corresponding Grassmannian cluster algebras are finite. As well as generating natural finite sets of letters, the tropical fans we discuss provide letters containing square roots. Remarkably, the minimal fan we consider provides all the square root letters recently discovered in an explicit two-loop eight-point NMHV calculation.

KeYwords: Differential and Algebraic Geometry, Scattering Amplitudes

ArXIV EPRINT: 1912.08217 


\section{Contents}

1 Introduction 1

2 Review of positive tropical $\operatorname{Gr}(4,8) \quad 2$

3 Infinite paths in $\operatorname{Gr}(4,8)$ and algebraic letters 8

4 Conclusions $\quad 15$

\section{Introduction}

Loop amplitudes in perturbative quantum field theory exhibit an intricate analytic structure. Understanding this structure in greater depth has allowed many advances and pushed the boundaries of what is computationally feasible by an enormous amount. In addition to the obvious practical benefits of a greater understanding, there has also been a surprising interplay with modern advances in mathematics, for example in the theory of polylogarithmic functions and their elliptic counterparts. In planar $\mathcal{N}=4$ super Yang-Mills theory there are many connections with various areas of mathematics, essentially because the theory is simple enough to allow for explicit results at higher orders in perturbation theory, while still being rich enough to exhibit many different analytic features.

Of particular relevance to this article is the connection between the singularities of planar loop amplitudes in $\mathcal{N}=4$ super Yang-Mills and cluster algebras related to Grassmannian spaces $\operatorname{Gr}(4, n)$. Cluster algebras were introduced and developed in [1-3] and are an area of intense ongoing research. Their relation to scattering amplitudes was first discussed in [4] in the context of on-shell diagrams. The connection to the branch cut singularities of amplitudes was established in [5] and explored further in e.g. [6]. This connection relates the cluster $\mathcal{A}$-coordinates of the cluster algebra with the symbol letters (potential logarithmic branch cuts) of the scattering amplitude. The cluster algebra connection explains the simple nine-letter alphabet of singularities previously found in sixparticle amplitudes [7] and it has been exploited in the context of the analytic bootstrap programme up to high loop orders [8-14]. Moreover the link to cluster algebras suggests a 42 letter alphabet for seven-particle amplitudes which has successfully been used to bootstrap amplitudes in [15-17].

Further support for the underlying connection to cluster algebras in the structure of scattering amplitudes comes from the discovery of cluster adjacency [18, 19]. This is an analytic property of amplitudes which relates different singularities to each other. In particular only cluster $\mathcal{A}$-coordinates which appear together in some cluster may appear in adjacent slots of the symbol. This property implies the Steinmann relations used in $[13,16]$ and their extended versions [20] (and under the assumption of physical branch cuts on the 
Euclidean sheet, seems also to be implied by them). An important point about the property of cluster adjacency is that it extends the role of the cluster algebra beyond the union of the $\mathcal{A}$-coordinates it generates; it also provides a role for the way the clusters themselves appear in the algebra. The property can also be phrased geometrically in terms of boundary facets of a polytope, only singularities corresponding to boundary components with the appropriate intersection can appear next to each other in the symbol. Pairs of letters corresponding to non-intersecting boundary components may not appear as neighbours.

Although the original connection to cluster algebras was inspired by the all-multiplicity result for two-loop MHV amplitudes in [21], it has been clear for some time that additional ingredients are needed when going beyond seven points. In the first instance the cluster algebras are finite type only for $\operatorname{Gr}(4,6)$ and $\operatorname{Gr}(4,7)$. For $\operatorname{Gr}(4,8)$ and beyond there are infinitely many cluster $\mathcal{A}$-coordinates, so some truncation to a finite set needs to be specified, as happens for the two-loop MHV amplitudes. Moreover at eight points and beyond there is an additional problem which is present already at one loop for $\mathrm{N}^{2} \mathrm{MHV}$ amplitudes. Four-mass box configurations appear which have letters which are not rational when expressed in terms of the Plücker coordinates for the Grassmannian spaces (i.e. in terms of momentum twistors [22]). Algebraic letters were also predicted for the two-loop NMHV amplitude [23, 24] by means of a Landau analysis (as initiated in this context in [25]) of the integrand provided by the amplituhedron [26, 27]. Letters containing square roots appear in the eight-point integrals considered in $[28,29]$. Recently, a two-loop NMHV calculation [30] based on solving the $\bar{Q}$-equation of [31, 32] for the dual octagonal (super) Wilson loop [33-37] has revealed a specific set of 18 multiplicatively independent algebraic letters in addition to 180 rational ones.

Here we propose that an answer to both problems may be provided by tropical geometry. Recently we investigated tropical fans associated to positive Grassmannians in [38]. This investigation was in part motivated by the connection made in [39] between tropical Grassmannians and scattering equations and their generalisations. In this context the tropical Grassmannian is related to a generalisation of tree-level biadjoint $\phi^{3}$ amplitudes. In the course of that investigation we highlighted the connection of the tropical geometry to the associated Grassmannian cluster algebra, a connection explored in part already in [40]. This connection will again play a central role in relating tropical fans to the singularities of scattering amplitudes, as we will discuss in the following.

Note added. Related topics on cluster algebras and scattering amplitudes are discussed in $[41,42]$.

\section{Review of positive tropical $\operatorname{Gr}(4,8)$}

Following the methods described by Speyer and Williams [40], in [38] we initiated a study of the fan describing the positive part of the tropical Grassmannian $\operatorname{Gr}(4,8)$. Here we will describe further features of the positive tropical Grassmannian $\operatorname{Gr}(4,8)$ which lead to the emergence of non-rational letters. Specifically, the $\operatorname{Gr}(4,8)$ cluster algebra is not finite, but of affine type $E_{7}^{(1,1)}$ [43]. This feature means that although the algebra is infinite, the 


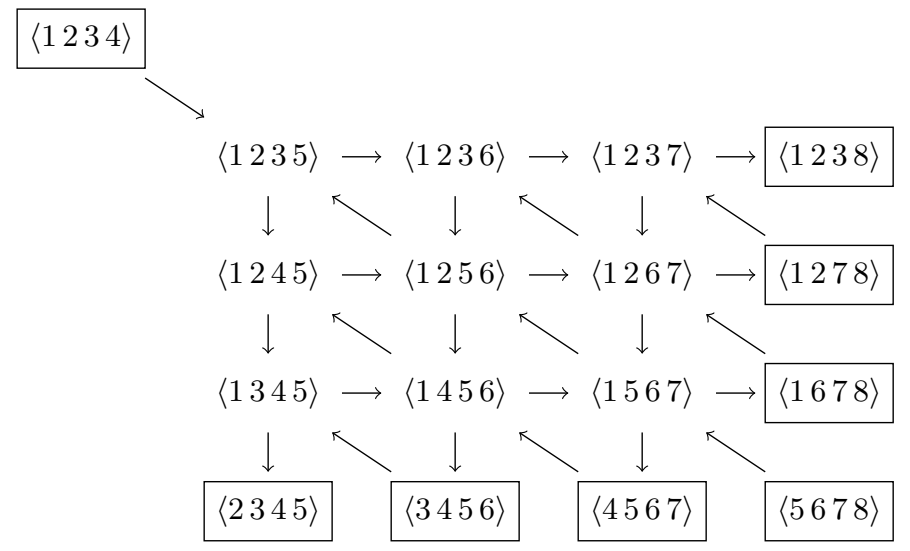

Figure 1. The initial cluster of the Grassmannian cluster algebra $\operatorname{Gr}(4,8)$.

infinity is controlled in a particular way and it makes $\operatorname{Gr}(4,8)$ a very natural example to consider in going beyond the finite cases. The affine nature of the cluster algebra leads us to natural infinite sequences of clusters which play a role in fully defining the Speyer-Williams fan (and related fans). Remarkably, the simplest infinite sequences lead to exactly the set of non-rational letters recently discovered in the two-loop eight-point NMHV amplitude [30].

Let us recall some of the basic features of cluster algebras associated to Grassmannians [44]. Our presentation will essentially follow that of [3], with the notation adapted to our conventions. A cluster algebra can be specified by some choice of initial cluster which can be encoded in a quiver diagram. The quiver diagram comprises a set of nodes, each labelled by a generator of the algebra called a cluster $\mathcal{A}$-coordinate. The nodes are either active or frozen and are connected by a network of arrows. The $\operatorname{Gr}(4,8)$ cluster algebra which is the focus of our interest here has an initial cluster of the form shown in figure 1 with $\mathcal{A}$-coordinates given by Plücker variables $\langle i j k l\rangle$. It has nine active nodes $a_{i}$ (labelled $1, \ldots, 9$ from the top left and descending column by column) and eight frozen nodes $f_{i}$ indicated by boxes making 17 nodes in total,

$$
\begin{aligned}
& \left\{a_{1}, \ldots, a_{9}\right\}=\{\langle 1235\rangle,\langle 1245\rangle,\langle 1345\rangle,\langle 1236\rangle,\langle 1256\rangle,\langle 1456\rangle,\langle 1237\rangle,\langle 1267\rangle,\langle 1567\rangle\}, \\
& \left\{f_{1}, \ldots, f_{8}\right\}=\{\langle 1234\rangle,\langle 2345\rangle,\langle 3456\rangle,\langle 4567\rangle,\langle 5678\rangle,\langle 1678\rangle,\langle 1278\rangle,\langle 1238\rangle\}
\end{aligned}
$$

When we need to consider all $17 \mathcal{A}$-coordinates together we order them as follows: $\left\{a_{1}, \ldots, a_{9}, f_{1}, \ldots, f_{8}\right\}$.

The arrows of the quiver diagram can be described by a square matrix $b$ (the exchange matrix) with entries

$$
b_{i j}=(\text { no. of arrows } i \rightarrow j)-(\text { no. of arrows } j \rightarrow i) .
$$

Here the matrix $b$ is skew-symmetric ${ }^{1}$ with indices running over all nodes (active and frozen) and in the case of $\operatorname{Gr}(4,8)$ therefore has dimension $(17 \times 17)$. We do not need to record arrows between frozen nodes so the bottom right $(8 \times 8)$ submatrix of $b$ is irrelevant in what follows.

\footnotetext{
${ }^{1}$ More generally in the study of cluster algebras it need only be skew-symmetrisable.
} 
In addition to the $\mathcal{A}$-coordinates and the $b$ matrix we have more data associated to the initial cluster. We also have a coefficient matrix, taken to be the $(9 \times 9)$ identity matrix. Additionally, to each active node $a_{i}$ we associate the $\mathbf{g}$-vector $\mathbf{e}_{i}$, the unit vector in the $i$ th direction.

Given the data for the initial cluster we may obtain the data for every other cluster by repeated mutation on active nodes. If we mutate on node $k$ we obtain a new $b$ matrix,

$$
b_{i j}^{\prime}= \begin{cases}-b_{i j} & \text { if } i=k \text { or } j=k . \\ b_{i j}+\left[-b_{i k}\right]_{+} b_{k j}+b_{i k}\left[b_{k j}\right]_{+} & \text {otherwise. }\end{cases}
$$

where $[x]_{+}=\max (x, 0)$. The $\mathcal{A}$-coordinate associated to the mutated node becomes

$$
a_{k}^{\prime}=\frac{1}{a_{k}} \prod_{i=1}^{m+n} a_{i}^{\left[b_{i k}\right]_{+}}+\prod_{i=1}^{m+n} a_{i}^{\left[-b_{i k}\right]_{+}}
$$

Note that, despite the denominator, the $\mathcal{A}$-coordinates can always be expressed as polynomials in Plücker coordinates after making use of Plücker relations. The coefficient matrix also transforms as follows, ${ }^{2}$

$$
c_{i j}^{\prime}= \begin{cases}-c_{i j} & \text { if } j=k . \\ c_{i j}-\left[-c_{i k}\right]_{+} b_{k j}+c_{i k}\left[-b_{k j}\right]_{+} & \text {otherwise. }\end{cases}
$$

Finally the $\mathbf{g}$-vector on node $k$ mutates as follows,

$$
\mathbf{g}_{k}^{\prime}=-\mathbf{g}_{k}+\sum_{i=1}^{n}\left[-b_{i k}\right]_{+} \mathbf{g}_{i}+\sum_{j=1}^{n}\left[c_{j k}\right]_{+} \mathbf{b}_{j}^{0}
$$

where $\mathbf{b}_{j}^{0}, j \in\{1, \ldots, 9\}$ corresponds to the $j$ th column of $b^{0}$, the exchange matrix for the initial cluster. By following the above rules one may obtain every cluster in the cluster algebra. In particular to each $\mathcal{A}$-coordinate generated there will be an associated $\mathbf{g}$-vector. For this reason we also use the notation $\mathbf{g}(a)$ for the $\mathbf{g}$-vector associated to the $\mathcal{A}$-coordinate $a$. As we described in [38] the g-vectors play a role in describing a tropical fan associated with the positive part of the tropical Grassmannian.

To describe the tropical fan of [40] we first introduce the cluster $\mathcal{X}$-coordinates. These may be obtained from the $\mathcal{A}$-coordinates of some cluster by writing for each active node $j$,

$$
x_{j}=\prod_{i=1}^{17} a_{i}^{b_{i j}}
$$

\footnotetext{
${ }^{2}$ Note that our conventions for the transformation of the coefficient matrix and the $\mathrm{g}$-vectors are modified with respect to those of Fomin and Zelevinsky [3] by replacing $b \rightarrow-b$.
} 
where the product ranges over all $\mathcal{A}$ coordinates (active and frozen). From the initial cluster we obtain a set of cluster $\mathcal{X}$-coordinates,

$$
\begin{aligned}
& x_{11}=\frac{\langle 1234\rangle\langle 1256\rangle}{\langle 1236\rangle\langle 1245\rangle} \quad x_{12}=\frac{\langle 1235\rangle\langle 1267\rangle}{\langle 1237\rangle\langle 1256\rangle} \quad x_{13}=\frac{\langle 1236\rangle\langle 1278\rangle}{\langle 1238\rangle\langle 1267\rangle} \\
& x_{21}=\frac{\langle 1235\rangle\langle 1456\rangle}{\langle 1256\rangle\langle 1345\rangle} \quad x_{22}=\frac{\langle 1236\rangle\langle 1245\rangle\langle 1567\rangle}{\langle 1235\rangle\langle 1456\rangle\langle 1267\rangle} \quad x_{23}=\frac{\langle 1237\rangle\langle 1256\rangle\langle 1678\rangle}{\langle 1236\rangle\langle 1567\rangle\langle 1278\rangle} \\
& x_{31}=\frac{\langle 1245\rangle\langle 3456\rangle}{\langle 1456\rangle\langle 2345\rangle} \quad x_{32}=\frac{\langle 1256\rangle\langle 1345\rangle\langle 4567\rangle}{\langle 1245\rangle\langle 3456\rangle\langle 1567\rangle} \quad x_{33}=\frac{\langle 1267\rangle\langle 1456\rangle\langle 5678\rangle}{\langle 1256\rangle\langle 4567\rangle\langle 1678\rangle},
\end{aligned}
$$

where we have chosen a labelling using a pair of indices for future convenience. This labelling is related to the usual labelling as follows

$$
\left\{x_{1}, \ldots, x_{9}\right\}=\left\{x_{11}, x_{21}, x_{31}, x_{12}, x_{22}, x_{32}, x_{13}, x_{23}, x_{33}\right\} .
$$

We may use the $\mathcal{X}$-coordinates $(2.8)$ to parametrise a $(4 \times 8)$ matrix $W$ (the web matrix) of the form

$$
W=\left(\mathbb{1}_{4} \mid M\right),
$$

where the $(4 \times 4)$ matrix $M$ has entries $m_{i j}$ given as a sum over Young tableaux of at most $(4-i)$ rows $\underline{\lambda}=\left\{\lambda_{1}, \ldots, \lambda_{4-i}\right\}$ with at most $(j-1)$ columns,

$$
m_{i j}=(-1)^{i} \sum_{\underline{\lambda} \in Y_{i j}} \prod_{k=1}^{4-i} \prod_{l=1}^{\lambda_{k}} x_{k l},
$$

where $Y_{i j}$ means the range $0 \leq \lambda_{4-i} \leq \ldots \leq \lambda_{1} \leq j-1$. The above formula is equivalent to the sum over paths of the web diagram described in [40].

The minors $\langle i j k l\rangle$, formed from the columns $i, j, k, l$ of the web matrix $W$ evaluate to polynomials in the cluster $\mathcal{X}$-coordinates $(2.8)$. They do so in such a way that the ratios of products of minors in (2.8) correctly evaluate to the $\mathcal{X}$-coordinates themselves. As examples of minors we find for instance

$$
\begin{aligned}
& \langle 1247\rangle=1+x_{11}+x_{11} x_{12}, \\
& \langle 2346\rangle=1+x_{11}+x_{11} x_{21}+x_{11} x_{21} x_{31} .
\end{aligned}
$$

To describe the positive tropical Grassmannian following [40] we evaluate these minors tropically. That is, we replace addition with minimum and multiplication with addition,

$$
\begin{aligned}
& \operatorname{Trop}\langle 1247\rangle=\min \left(0, \tilde{x}_{11}, \tilde{x}_{11}+\tilde{x}_{12}\right), \\
& \operatorname{Trop}\langle 2346\rangle=\min \left(0, \tilde{x}_{11}, \tilde{x}_{11}+\tilde{x}_{21}, \tilde{x}_{11}+\tilde{x}_{21}+\tilde{x}_{31}\right),
\end{aligned}
$$

where we remind the reader that these are tropical polynomials by using $\tilde{x}$ instead of $x$. Each tropical minor defines a number of regions (each one a cone) of piecewise linearity in the $\tilde{x}$ space. Taking all tropical minors together we get many such regions whose overlap defines a fan. Each maximal cone of the fan is a region in which all tropical minors are linear functions. If we intersect the fan with the unit sphere in the (nine-dimensional) space of the $\tilde{x}$, each maximal cone becomes an eight-dimensional facet of a polyhedral complex. 
The boundaries of the facets are locations where at least one minor is between two different regions of piecewise linearity. For example, the minor $\operatorname{Trop}\langle 1247\rangle$ in (2.13) has boundaries between regions of piecewise linearity if one of the following tropical hypersurface conditions holds,

$$
\begin{aligned}
\tilde{x}_{11} & =0 \leq \tilde{x}_{11}+\tilde{x}_{12} \\
\text { or } \quad \tilde{x}_{11}+\tilde{x}_{21} & =0 \leq \tilde{x}_{11} \\
\text { or } \quad \tilde{x}_{11} & =\tilde{x}_{11}+\tilde{x}_{22} \leq 0 .
\end{aligned}
$$

Each eight-dimensional facet has seven-dimensional boundaries where one such condition is obeyed. The boundaries themselves have six-dimensional boundaries where two linearly independent equalities and the associated inequalities are obeyed. Proceeding in this way we arrive at zero-dimensional boundaries, called rays, where eight linearly independent tropical hypersurface conditions are obeyed.

In fact one may generalise the above discussion and consider multiple different tropical fans associated to a given Grassmannian. We could consider a fan defined by only a subset of minors, for example only those minors of the form $\langle i i+1 j j+1\rangle$ or $\langle i-1 i i+1 j\rangle$. Or we could refine the fan further by including tropical evaluations of cluster $\mathcal{A}$-coordinates which are polynomials in minors, as well as the minors themselves. More generally we will define a fan by choosing some subset $\mathcal{S}$ of tropically evaluated $\mathcal{A}$-coordinates and we denote the fan by $F(\mathcal{S})$. The fan of Speyer and Williams described above then corresponds to the choice where $\mathcal{S}$ is the set of all minors.

It is important to emphasise that for any given choice of the set $\mathcal{S}$, the resulting fan is finite and in particular has a finite number of rays. One may systematically solve the tropical hypersurface conditions to find all the rays of some fan $F(\mathcal{S})$. In [38] we described another approach which makes use of the associated cluster algebra. In the case of finite cluster algebras, including the finite Grassmannian algebras studied in [38], the cluster algebra also defines a fan by means of its $\mathbf{g}$-vectors. In fact the $\mathbf{g}$-vector fan coincides with the fan obtained by considering $\mathcal{S}$ to be given by the set of all $\mathcal{A}$-coordinates (not just all minors). It is therefore in general a refinement of the Speyer-Williams fan. In the case of $\operatorname{Gr}(2, n)$ the $\mathcal{A}$-coordinates are all minors and the $\mathbf{g}$-vector fan coincides with the Speyer-Williams fan.

In the case of the Grassmannian $\operatorname{Gr}(4,8)$ we cannot immediately define a fan using all the cluster $\mathcal{A}$-coordinates since there are infinitely many. We can nevertheless use the g-vectors of the cluster algebra as candidate rays of any fan $F(\mathcal{S})$ defined by tropical evaluation of a finite set $\mathcal{S}$ of cluster $\mathcal{A}$-coordinates. If we restrict ourselves to looking for rays, this approach is very effective. Systematically constructing the rays of the fan can be quite cumbersome for large fans but, given a candidate ray, it is trivial to check if it is truly a ray. As we already outlined in [38], if we consider the Speyer-Williams fan where we take $\mathcal{S}$ to be the set of all minors then we find that $356 \mathrm{~g}$-vectors of the cluster algebra are also rays of the fan.

We can similarly determine that for $\mathcal{S}=\{\langle i i+1 j j+1\rangle,\langle i-1, i, i+1, j\rangle\}$ (the maximal parity-invariant subset of minors) we find that $272 \mathrm{~g}$-vectors are rays. For 


\begin{tabular}{|c|cc|}
\hline $\mathcal{S}$ & g-vector rays & limit rays \\
\hline$\{\langle i i+1 j j+1\rangle,\langle i-1 i i+1 j\rangle\}$ & 272 & 2 \\
$\{\langle i j k l\rangle\}$ & 356 & 4 \\
$\{\langle i j k l\rangle,\langle\overline{i j k l}\rangle\}$ & 544 & 4 \\
\hline
\end{tabular}

Table 1. Number of rays of the fans $F(\mathcal{S})$ for different choices of $\mathcal{S}$.

$\mathcal{S}=\{\langle i j k l\rangle,\langle\overline{i j k l}\rangle\}$ (the parity completion of all minors) we find that 544 g-vectors are rays. Passing from the cluster algebra to a choice of fan defined by a set $\mathcal{S}$ of $\mathcal{A}$ coordinates is therefore a natural way to obtain a finite truncation of the infinite set of cluster $\mathcal{A}$ coordinates.

Most interestingly, in none of the above cases do the $\mathbf{g}$-vectors provide a complete set of rays. In fact we find additional rays which complete the above sets of $\mathbf{g}$-vectors as shown in table 1. As we will describe in the next section, the cluster algebra can also be used to find the extra rays which are not g-vectors. In fact they arise as limits of special infinite sequences of $\mathbf{g}$-vectors so we refer to them as limit rays.

To each $\mathbf{g}$-vector is associated a cluster $\mathcal{A}$-coordinate. We will conclude this section by explicitly listing the $\mathcal{A}$-coordinates corresponding to the $272 \mathrm{~g}$-vector rays in the least refined fan described in table 1 . If we also include the eight frozen $\mathcal{A}$-coordinates then the resulting $280 \mathcal{A}$ coordinates contain the 196 rational letters found in [24] as an alphabet predicted by Landau analysis for the two-loop NMHV amplitude. In fact the explicit result for the two-loop octagon found recently in [30] contains only 180 of these rational letters. In addition the two-loop NMHV octagon contains 18 multiplicatively independent algebraic letters involving square roots, only four of which (corresponding to the letters of the possible four-mass box integral topologies) are contained in the list in [24].

We begin the list of 280 letters (including 8 frozen) by recalling the 196 rational letters of [24],

- 68 four-brackets of the form $\langle a a+1 b c\rangle$,

- 8 cyclic images of $\langle 12 \overline{4} \cap \overline{7}\rangle$,

- 40 cyclic images of $\langle 1(23)(45)(78)\rangle,\langle 1(23)(56)(78)\rangle,\langle 1(28)(34)(56)\rangle,\langle 1(28)(34)(67)\rangle$, $\langle 1(28)(45)(67)\rangle$

- 48 dihedral images of $\langle 1(23)(45)(67)\rangle,\langle 1(23)(45)(68)\rangle,\langle 1(28)(34)(57)\rangle$,

- 8 cyclic images of $\langle\overline{2} \cap(245) \cap \overline{8} \cap(856)\rangle$,

- 8 distinct images of $\langle\overline{2} \cap(245) \cap \overline{6} \cap(681)\rangle$,

- 16 dihedral images of $\langle 12345678\rangle$.

In addition, we have the following 84 rational letters,

- 2 letters, $\langle 1357\rangle$ and $\langle 2468\rangle$,

- 8 cyclic images of $\langle 1(23)(46)(78)\rangle$ (this set is closed under reflections), 
- 16 dihedral images of $\langle 1(27)(34)(56)\rangle$,

- 2 cyclic images of $\langle\overline{2} \cap \overline{4} \cap \overline{6} \cap \overline{8}\rangle$ (this set returns to itself under two rotations and it is closed under reflections),

- 8 cyclic images of $\langle\overline{2} \cap(246) \cap \overline{6} \cap \overline{8}\rangle$ (this set is closed under reflections),

- 32 dihedral images of $\langle\langle 12435678\rangle\rangle,\langle\langle 12436578\rangle$,

- 16 dihedral images of $\langle 1234\rangle\langle 1678\rangle\langle 2456\rangle-\langle 1267\rangle\langle 1348\rangle\langle 2456\rangle+\langle 1248\rangle\langle 1267\rangle\langle 3456\rangle$.

In the above we have defined $\langle\langle a b c d e f g h\rangle=\langle a b c d\rangle\langle a b e f\rangle\langle\operatorname{degh}\rangle-\langle a b d e\rangle\langle a b e f\rangle\langle c d g h\rangle+$ $\langle a b d e\rangle\langle a b g h\rangle\langle c d e f\rangle$.

In an ancillary file we list the g-vectors and their corresponding letters for the first two cases of table 1.

We now turn to describing the extra rays obtained by limits of infinite sequences and the resulting algebraic letters.

\section{Infinite paths in $\operatorname{Gr}(4,8)$ and algebraic letters}

As we have seen in the previous discussion, the relation between amplitude singularities and cluster algebra data requires some refinement when going beyond seven points. In the first instance, the two-loop NMHV octagon has algebraic letters which do not correspond to any cluster $\mathcal{A}$-coordinate. In addition, in truncating the infinite set of $\mathcal{A}$-coordinates by considering some tropical fan $F(\mathcal{S})$ as described above, the rays of $F(\mathcal{S})$ are not all described by $\mathrm{g}$-vectors of the cluster algebra.

We may address both of the above difficulties by realising that the infinite number of clusters can usefully be organised into infinite sequences, each of which can be related to an infinite rank two cluster algebra with two nodes and a doubled arrow between them. Such algebras were considered in e.g. [45] and it was already noted there that under repeated mutation the $\mathbf{g}$-vectors asymptote to a limiting vector. In fact, in the affine case which is relevant here, the same limiting vector can be obtained by repeated mutation with either choice of initial node (i.e. both directions asymptote to the same limit vector).

If we ignore the frozen nodes (and ignore the values of the $\mathcal{A}$-coordinates on the active nodes) there are 506 distinct quiver diagrams that arise in the $\operatorname{Gr}(4,8)$ cluster algebra. The fact that there are only finitely many is a feature of the affine cases of Grassmannian cluster algebras $\operatorname{Gr}(4,8)$ and $\operatorname{Gr}(3,9)$ and these algebras are referred to as finite mutation type. Out of the 506 quivers, 491 have only single arrows while 15 have a doubled arrow. These latter type have the shape of the $E_{7}^{(1,1)}$ quiver diagram shown in figure 2, or one related to it by mutation in the $A_{2} \times A_{2}$ subalgebra generated by mutations on the $a_{i}$ type nodes [43].

Each diagram of the form of figure 2 forms part of a doubly infinite rank-two affine sequence, generated by alternating mutations on the $w_{0}$ and $z_{0}$ nodes. In each such sequence we can find some cluster (actually an $A_{2} \times A_{2}$ subalgebra of clusters) in which the frozen nodes are all outgoing from $w_{0}$ and incoming to $z_{0}$. We illustrate this by a simplified 


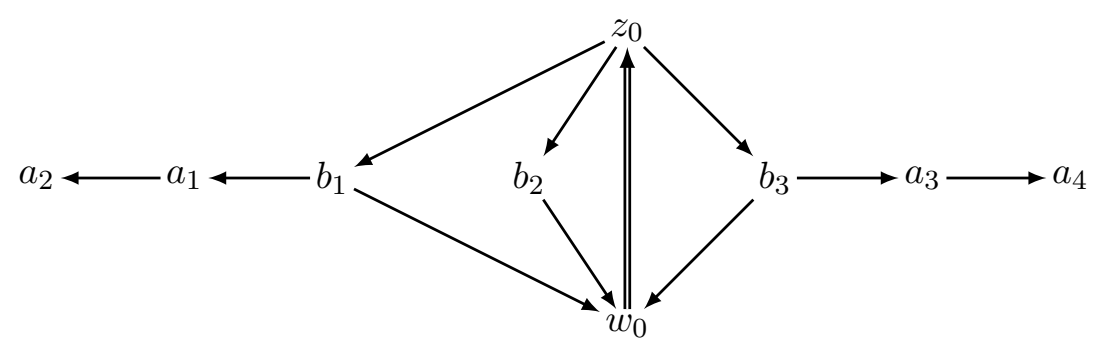

Figure 2. The $E_{7}^{(1,1)}$ shaped clusters with a doubled arrow between two cluster $\mathcal{A}$-coordinates, $w_{0}$ and $z_{0}$. By mutation on the $a_{i}$ nodes we generate an $A_{2} \times A_{2}$ subalgebra of clusters containing the same $w_{0}, z_{0}$ and $b_{i}$ nodes. Frozen nodes are omitted here.

diagram which we refer to as an origin cluster where we ignore the $a_{i}$ nodes, combine the three $b_{i}$ nodes into a single node,

$$
b=b_{1} b_{2} b_{3},
$$

and combine all frozen nodes outgoing from $w_{0}$ into $f_{w}$ and those incoming to $z_{0}$ into $f_{z}$,

$$
f_{w}=\prod_{i=1}^{8} f_{i}^{m_{i}}, \quad f_{z}=\prod_{i=1}^{8} f_{i}^{n_{i}}, \quad m_{i}, n_{i} \in \mathbb{N}_{0} .
$$

Such a simplified diagram is illustrated at the top of figure 3 .

The initial mutations to generate the infinite double sequence take the form

$$
\begin{aligned}
& z_{1} w_{0}=b+f_{w} z_{0}^{2}, \\
& w_{1} z_{0}=b+f_{z} w_{0}^{2} .
\end{aligned}
$$

Thereafter the mutations in the $z$-direction and $w$-direction take the uniform form for $n \geq 0$,

$$
\begin{aligned}
z_{n+2} z_{n} & =\mathcal{C} \mathcal{F}^{n}+z_{n+1}^{2}, \\
w_{n+2} w_{n} & =\tilde{\mathcal{C}} \mathcal{F}^{n}+w_{n+1}^{2} .
\end{aligned}
$$

The coefficients $\mathcal{C}$ and $\tilde{\mathcal{C}}$ are given by

$$
\begin{aligned}
& \mathcal{C}=b f_{z}, \\
& \tilde{\mathcal{C}}=b f_{w},
\end{aligned}
$$

while the factor $\mathcal{F}$ is the product over the frozen nodes,

$$
\mathcal{F}=f_{w} f_{z} .
$$

The transformations of the g-vectors while performing the doubly infinite sequence of mutations are very simple. After a few initial mutations the differences in consecutive g-vectors stabilise and we arrive at the form

$$
\mathbf{g}\left(z_{n+1}\right)-\mathbf{g}\left(z_{n}\right)=\mathbf{g}\left(w_{0}\right)-\mathbf{g}\left(z_{0}\right)=\mathbf{g}\left(w_{n+1}\right)-\mathbf{g}\left(w_{n}\right)
$$




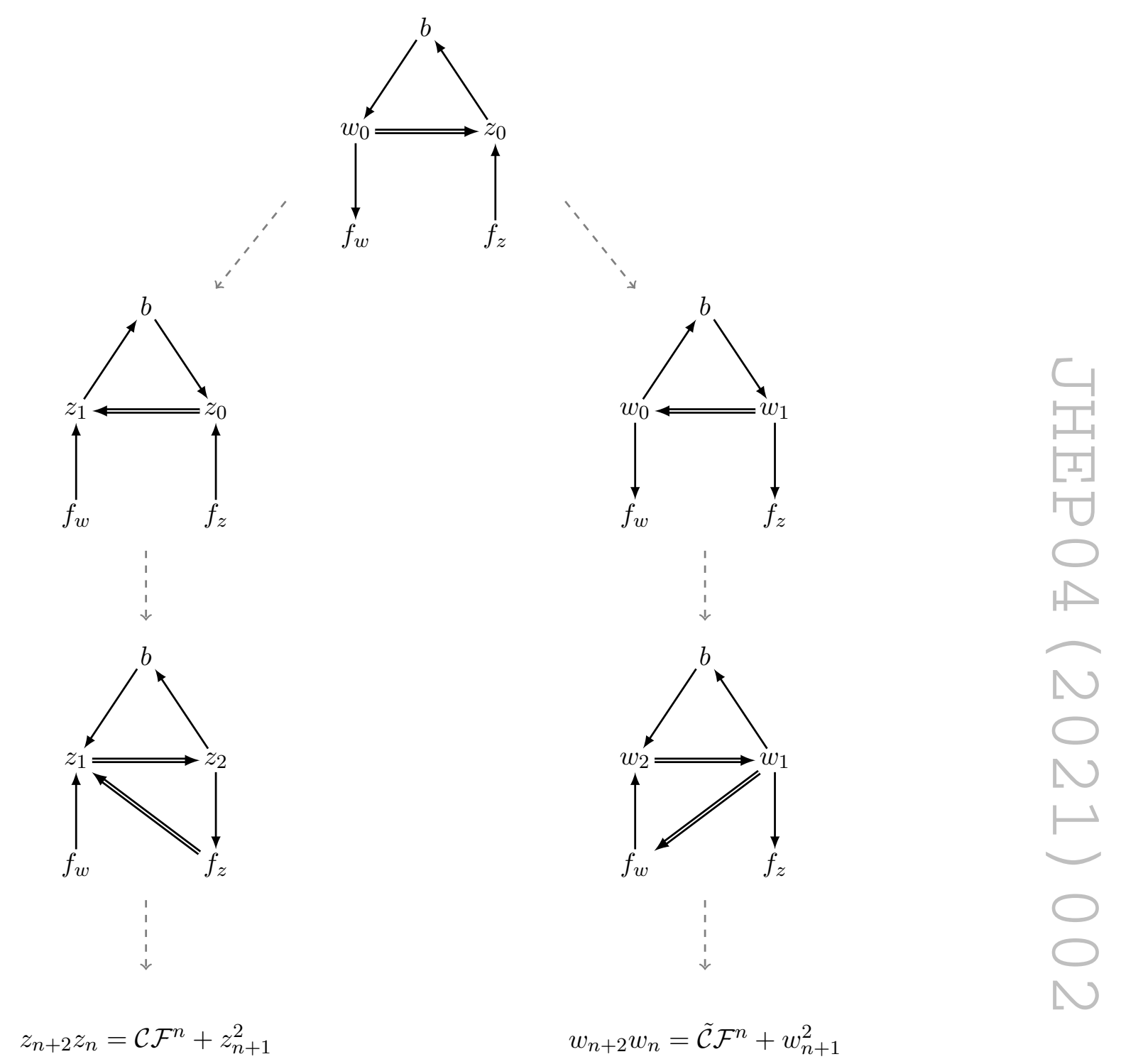

Figure 3. The doubly infinite sequence corresponding to the embeddings of the affine $A_{2}$ cluster algebra into the $\operatorname{Gr}(4,8)$ cluster algebra. After mutating one step from the origin cluster on either node, the repeated mutations give rise to a regular recurrence relation.

This shows that in either direction the g-vectors will asymptote to the limit ray

$$
\mathbf{g}_{\infty}=\mathbf{g}\left(w_{0}\right)-\mathbf{g}\left(z_{0}\right)
$$

In fact we find many different origin clusters of the form shown at the top of figure 3, with different $w_{0}$ and $z_{0}$ (and hence different $\mathbf{g}\left(w_{0}\right)$ and $\mathbf{g}\left(z_{0}\right)$ ) but with the same limit ray $\mathbf{g}_{\infty}$.

We may recast the quadratic recurrence relations (3.4) in a matrix form,

$$
\left(\begin{array}{cc}
z_{n+2} & z_{n+1} \\
z_{n+1} & z_{n}
\end{array}\right)=\left(\begin{array}{cc}
z_{n+1} & z_{n} \\
z_{n} & z_{n-1}
\end{array}\right)\left(\begin{array}{cc}
\mathcal{P} & 1 \\
-\mathcal{F} & 0
\end{array}\right)=\left(\begin{array}{cc}
z_{2} & z_{1} \\
z_{1} & z_{0}
\end{array}\right)\left(\begin{array}{cc}
\mathcal{P} & 1 \\
-\mathcal{F} & 0
\end{array}\right)^{n}
$$


and similarly for $z \rightarrow w$. Taking the determinant of the matrix relations (3.9) yields the original quadratic relations (3.4) since

$$
z_{2} z_{0}=\mathcal{C}+z_{1}^{2} .
$$

We may verify that the matrix recursion (3.9) consistently generates the same sequence $z_{n}$ as the quadratic recursion (3.4) provided that $\mathcal{P}$ obeys

$$
z_{2}=z_{1} \mathcal{P}-z_{0} \mathcal{F}
$$

Hence we require that $\mathcal{P}$ is related to $\mathcal{C}$ via

$$
\mathcal{C}+z_{1}^{2}+z_{0}^{2} \mathcal{F}=z_{0} z_{1} \mathcal{P}
$$

Remarkably, $\mathcal{P}$ can be shown to be a polynomial, that is we can find a factor of $z_{0} z_{1}$ within the combination on the l.h.s. of (3.12). If we write $\mathcal{C}$ and $\mathcal{F}$ in terms of the cluster $\mathcal{A}$-coordinates of the origin cluster we find

$$
\begin{aligned}
\mathcal{C}+z_{1}^{2}+z_{0}^{2} \mathcal{F} & =b f_{z}+z_{1}^{2}+z_{0}^{2} f_{w} f_{z}, \\
& =z_{1}\left(f_{z} w_{0}+z_{1}\right),
\end{aligned}
$$

where the second step is achieved by using the first relation in (3.3) to eliminate $b$. We have made the factor of $z_{1}$ manifest and it remains to show that there is also a factor of $z_{0}$ in the remaining combination $\left(f_{z} w_{0}+z_{1}\right)$. To show this we consider instead the square of this combination,

$$
\begin{aligned}
\left(f_{z} w_{0}+z_{1}\right)^{2} & =f_{z}^{2} w_{0}^{2}+2 f_{z} w_{0} z_{1}+z_{1}^{2} \\
& =f_{z}\left(w_{1} z_{0}-b\right)+2 f_{z}\left(b+f_{w} z_{0}^{2}\right)+\left(z_{2} z_{0}-b f_{z}\right) \\
& =z_{0}\left(f_{z} w_{1}+2 f_{z} f_{w} z_{0}+z_{2}\right)
\end{aligned}
$$

In the second step we have used the relations (3.3) and the quadratic recurrence formula (3.4) for $z$ in the case $n=2$. We have succeeded in finding a factor of $z_{0}$ in the square factor, but since all quantities involved are polynomials in Plücker coordinates, it must be that the original factor without the square also has a factor of $z_{0}$. Hence we conclude that

$$
\mathcal{P}=\frac{f_{z} w_{0}+z_{1}}{z_{0}}
$$

is a polynomial even if this property is not manifest from the above equation. By considering the $w_{n}$ sequence instead we arrive at an equivalent formula for $\mathcal{P}$,

$$
\mathcal{P}=\frac{f_{w} z_{0}+w_{1}}{w_{0}} .
$$

Note that both $\mathcal{P}$ and $\mathcal{F}$ are invariant under swapping the $z$ sequence and the $w$ sequence (along with swapping $f_{z}$ with $f_{w}$ ). Note also that $\mathcal{P}$ is manifestly positive in the region where all $\mathcal{A}$-coordinates are positive. 
Returning to the matrix recursion we see that it is equivalent to a linear recursion formula

$$
z_{n+2}=z_{n+1} \mathcal{P}-z_{n} \mathcal{F},
$$

of which (3.11) is just the first case. Of course we also have the same recursion formula for the $w_{n}$. Once a polynomial form for $\mathcal{P}$ is obtained, this linear recursion formula provides a manifestly polynomial form for all the $z_{n}$ cluster coordinates (and similarly the $w_{n}$ ). Note that the linear recursion would just be the Fibonacci recursion relation if we had $\mathcal{P}=-\mathcal{F}=1$. The linear recursion formula is neatly solved by the following generating function

$$
G_{z}(x)=\frac{z_{1}-z_{0} \mathcal{F} x}{1-\mathcal{P} x+\mathcal{F} x^{2}}=\sum_{n=0}^{\infty} z_{n+1} x^{n},
$$

and similarly for $w \leftrightarrow z$. It follows immediately that the asymptotic limit of the ratios of the $z_{n}$ is controlled by the roots of the quadratic in the denominator,

$$
\lim _{n \rightarrow \infty} \frac{z_{n}}{z_{n-1}}=\mathcal{P}+\sqrt{\Delta}, \quad \Delta=\mathcal{P}^{2}-4 \mathcal{F} .
$$

Using this fact we can write an explicit form for the $z_{n}$,

$$
z_{n}=\frac{1}{2^{n+1}}\left[\left(z_{0}+B_{z} \sqrt{\Delta}\right)(\mathcal{P}+\sqrt{\Delta})^{n}+\left(z_{0}-B_{z} \sqrt{\Delta}\right)(\mathcal{P}-\sqrt{\Delta})^{n}\right]
$$

with $B_{z}$ defined by

$$
B_{z}=\frac{2 z_{1}-z_{0} \mathcal{P}}{\Delta}
$$

We have a similar formula for the $w_{n}$ sequence obtained by swapping $z \leftrightarrow w$ everywhere. For a sequence of mutations generating $\mathbf{g}$-vectors which asymptote to a given limit ray $\mathbf{g}_{\infty}$, we find that $\mathcal{P}$ and $\mathcal{F}$ (and hence the limit of the ratio (3.19)) depend only on the limit ray. The actual path towards the limit (and therefore the $z_{n}$ or $w_{n}$ ) is distinguished by the values of $z_{0}$ and $z_{1}$ (or $w_{0}$ and $w_{1}$ ).

In the limit of large $n$, the term with $(\mathcal{P}+\sqrt{\Delta})^{n}$ dominates over the term $(\mathcal{P}-\sqrt{\Delta})^{n}$. Its coefficient $\left(z_{0}+B_{z} \sqrt{\Delta}\right)$ depends on the path of approach to the limit. Since the product $\left(z_{0}+B_{z} \sqrt{\Delta}\right)\left(z_{0}-B_{z} \sqrt{\Delta}\right)$ is rational ${ }^{3}$ we identify the ratio

$$
\phi_{z}=\frac{z_{0}+B_{z} \sqrt{\Delta}}{z_{0}-B_{z} \sqrt{\Delta}}=\frac{z_{0} \mathcal{P}-2 f_{z} w_{0}+z_{0} \sqrt{\Delta}}{z_{0} \mathcal{P}-2 f_{z} w_{0}-z_{0} \sqrt{\Delta}}
$$

with a new algebraic letter associated to the path. We also have a letter obtained from the limit of the $w$ sequence whose formula is the same except for swapping $z \leftrightarrow w$ everywhere,

$$
\phi_{w}=\frac{w_{0}+B_{w} \sqrt{\Delta}}{w_{0}-B_{w} \sqrt{\Delta}}=\frac{w_{0} \mathcal{P}-2 f_{w} z_{0}+w_{0} \sqrt{\Delta}}{w_{0} \mathcal{P}-2 f_{w} z_{0}-w_{0} \sqrt{\Delta}} .
$$

Note that we have many origin clusters, each of which provides two paths (the $z$ branch and the $w$ branch) towards the same limit ray $\mathbf{g}_{\infty}$. The square root $\sqrt{\Delta}$ which appears will

\footnotetext{
${ }^{3}$ For the cases we consider shortly, it is always a multiplicative combination of the 280 rational letters given in at the end of section 2 .
} 
be common for all algebraic letters coming from a given limit. Only the rational coefficients (determined by the data of the origin cluster) will depend on the actual path.

Let us recall that the smallest fan from those listed in table 1 has two limit rays in addition to the $272 \mathrm{~g}$-vector rays. For the case of a path that asymptotes to the first limit ray we find

$$
\begin{aligned}
\mathbf{g}_{\infty}^{(1)} & =(1,-1,0,-1,0,1,0,1,-1), \\
\mathcal{P} & =\langle 1256\rangle\langle 3478\rangle-\langle 1278\rangle\langle 3456\rangle-\langle 1234\rangle\langle 5678\rangle, \\
\mathcal{F} & =\langle 1234\rangle\langle 3456\rangle\langle 5678\rangle\langle 1278\rangle .
\end{aligned}
$$

while the second limit ray

$$
\mathbf{g}_{\infty}^{(2)}=(0,1,0,1,0,-1,0,-1,0)
$$

has $\mathcal{P}$ and $\mathcal{F}$ related to those in (3.24) by a cyclic rotation by one unit. The precise values of $z_{0}$ and $z_{1}$ (or $w_{0}$ and $w_{1}$ ) depend on the path of approach.

We find 64 origin clusters whose associated limit rays are either $\mathbf{g}_{\infty}^{(1)}$ or $\mathbf{g}_{\infty}^{(2)}$ described above. Among them are four clusters with the nodes $w_{0}$ and $z_{0}$ connected by the doubled arrow given by

$$
\langle j(12)(i k)(78)\rangle \Longrightarrow\langle 12 i j\rangle
$$

where $i \in\{3,4\}$ and $(j, k)$ is a permutation of $(5,6)$. Each origin cluster with the rank two affine subalgebras of the form (3.26) has the limit ray $\mathbf{g}_{\infty}^{(1)}$ and frozen nodes given by

$$
f_{z}=f_{1}=\langle 1234\rangle, \quad f_{w}=f_{3} f_{5} f_{7}=\langle 3456\rangle\langle 5678\rangle\langle 1278\rangle,
$$

in agreement with eq. (3.24). The four origin clusters are listed in table 2 along with the data from the original cluster diagram that they come from, including the $b$ nodes and the $A_{2} \times A_{2}$ subalgebra generated by the $a_{i}$ type nodes of figure 2 . The full set of 64 origin clusters whose limit rays are $\mathbf{g}_{\infty}^{(1)}$ or $\mathbf{g}_{\infty}^{(2)}$ are then obtained from the four described in (3.26) by dihedral transformations.

Each origin cluster produces two algebraic letters $\phi_{z}$ and $\phi_{w}$ defined by eqs. (3.22) and (3.23). Thus we have a set of 128 algebraic letters associated to the two limit rays $\mathbf{g}_{\infty}^{(1)}$ and $\mathbf{g}_{\infty}^{(2)}$. Each limit ray is therefore associated with significantly more data than any $\mathbf{g}$ vector ray, each of which is associated to a single rational letter. The 128 letters associated to $\mathbf{g}_{\infty}^{(1)}$ and $\mathbf{g}_{\infty}^{(2)}$ are not all multiplicatively independent and remarkably they generate the same space as the 18 multiplicatively independent algebraic letters found in [30]! The two-loop NHMV eight-point amplitude is therefore consistent with the data obtained from the smallest fan in table 1 in that the associated alphabet is covered by the rays of the fan.

The set of 128 algebraic letters described above is closed under parity, as the doubly infinite sequences themselves map to each other under parity. The origin clusters themselves do not necessarily map to origin clusters but sometimes map to an adjacent cluster in the infinite sequence. In an ancillary file we explicitly list the 128 algebraic letters.

The other fans in table 1 have four limit rays. These are similarly associated to their own set of origin clusters, again 64 such clusters, each generating two algebraic letters 


\begin{tabular}{|c|c|c|}
\hline Sub affine $A_{2}: w_{0} \Longrightarrow z_{0}$ & $b=b_{1} b_{2} b_{3}$ & Residual $A_{2} \times A_{2}$ \\
\hline$\langle 5(12)(36)(87)\rangle \Longrightarrow\langle 1235\rangle$ & $\begin{array}{l}\langle 1256\rangle \\
\times\langle 3(12)(56)(78)\rangle \\
\times\langle 5(12)(34)(78)\rangle\end{array}$ & $\begin{array}{l}\langle 1345\rangle \rightarrow\langle 1346\rangle \\
\langle 1237\rangle \rightarrow\langle 1247\rangle\end{array}$ \\
\hline$\langle 6(12)(35)(78)\rangle \Longrightarrow\langle 1236\rangle$ & $\begin{array}{l}\langle 1256\rangle \\
\times\langle 3(12)(56)(78)\rangle \\
\times\langle 6(12)(34)(78)\rangle\end{array}$ & $\begin{array}{l}\langle 1345\rangle \rightarrow\langle 1346\rangle \\
\langle 1237\rangle \longrightarrow\langle 3567\rangle\end{array}$ \\
\hline$\langle 5(12)(46)(87)\rangle \Longrightarrow\langle 1245\rangle$ & $\begin{array}{l}\langle 1256\rangle \\
\times\langle 4(12)(56)(78)\rangle \\
\times\langle 5(12)(34)(78)\rangle\end{array}$ & $\begin{array}{l}\langle 1237\rangle \longrightarrow\langle 1247\rangle \\
\langle 1237\rangle \longrightarrow\langle 1247\rangle\end{array}$ \\
\hline$\langle 6(12)(45)(78)\rangle \Longrightarrow\langle 1246\rangle$ & $\begin{array}{l}\langle 1256\rangle \\
\times\langle 4(12)(56)(78)\rangle \\
\times\langle 6(12)(34)(78)\rangle\end{array}$ & 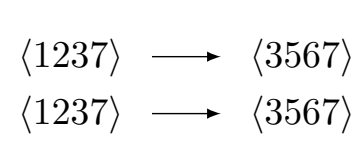 \\
\hline
\end{tabular}

Table 2. Four types of clusters that act as origins of doubly-infinite sequences.

according to (3.22) and (3.23). In this case the $\mathcal{P}$ and $\mathcal{F}$ associated to $\mathbf{g}_{\infty}^{(3)}$ are as follows,

$$
\begin{aligned}
\mathbf{g}_{\infty}^{(3)}= & (-1,0,1,0,2,-1,1,-1,-1), \\
\mathcal{P}= & \langle 1237\rangle\langle 1458\rangle\langle 2468\rangle\langle 3567\rangle-\langle 1238\rangle\langle 1567\rangle\langle 2468\rangle\langle 3457\rangle \\
& -\langle 1238\rangle\langle 1678\rangle\langle 2345\rangle\langle 4567\rangle-\langle 1237\rangle\langle 1358\rangle\langle 2468\rangle\langle 4567\rangle \\
& -\langle 1234\rangle\langle 1278\rangle\langle 3456\rangle\langle 5678\rangle, \\
\mathcal{F}= & \langle 1234\rangle\langle 2345\rangle\langle 3456\rangle\langle 4567\rangle\langle 5678\rangle\langle 1678\rangle\langle 1278\rangle\langle 1238\rangle .
\end{aligned}
$$

The $\mathcal{P}$ associated to the other limit ray

$$
\mathbf{g}_{\infty}^{(4)}=(1,1,-1,1,-2,0,-1,0,1),
$$

is related to that in (3.28) by a cyclic rotation by one unit while the $\mathcal{F}$ is the same (note that $\mathcal{F}$ in (3.28) is the product of all frozen $\mathcal{A}$-coordinates and therefore is cyclic invariant). The algebraic letters associated to the limit rays $\mathbf{g}_{\infty}^{(3)}$ and $\mathbf{g}_{\infty}^{(4)}$ are therefore of a different nature with different square roots. So far we do not have any example of an amplitude where they appear. They might appear at higher loop orders in eight-point amplitudes than are currently known explicitly.

We should also stress that there are more origin clusters (infinitely many) each of which has its own limit vector associated to it and its own type of square roots. However the limit vectors obtained are not rays of any of the fans listed in table 1 . One could imagine making yet more refined fans $F(\mathcal{S})$ by taking yet larger sets $\mathcal{S}$ of $\mathcal{A}$-coordinates to define them. It is possible that the other limit vectors beyond the four described above become rays of such fans. 


\section{Conclusions}

The fact that we find exactly the same letters appearing in [30] from tropical geometry and cluster algebras is very exciting. Ultimately we must remember that the tropical problems we have been considering here arise purely from kinematics. Momentum twistors provide a natural unconstrained set of coordinates for the kinematical space of colourordered amplitudes in the planar limit and dual conformal symmetry [46] dictates that we should consider $s l_{4}$ invariant combinations of them. This leads directly to the association of the Grassmannian $\operatorname{Gr}(4, n)$, or more precisely $\operatorname{Conf}_{n}\left(\mathbb{P}^{3}\right)=\operatorname{Gr}(4, n) /\left(\mathbb{C}^{*}\right)^{n-1}$, to the kinematic space of massless scattering in planar $\mathcal{N}=4$ super Yang-Mills theory. The dual conformally invariant (or $s l_{4}$ invariant) quantities are the Plücker coordinates $\langle i j k l\rangle$ and they obey quadratic Plücker relations, for example the following,

$$
\langle i j k[l\rangle\langle m n p q]\rangle=0 \text {. }
$$

Tropicalising such polynomial relations gives the tropical Grassmannian as considered by Speyer and Sturmfels [47]. Considering its positive part leads to the tropical fans of Speyer and Williams [40]. As we have discussed, these have a direct connection to the Grassmannian cluster algebras. The g-vectors of the cluster algebras provide rays for the tropical fans, even in the case where the algebra is not finite, such as the case studied here $\operatorname{Gr}(4,8)$. To these rays are associated rational $\mathcal{A}$-coordinates which play the role of symbol letters characterising the singularities of the polylogarithmic functions describing the scattering amplitudes. Moreover the additional rays of the fan arise as limits of natural infinite sequences of $\mathbf{g}$-vectors. To these are associated sets of algebraic letters involving square roots.

It remains to clarify which fans correspond to which amplitudes. We have seen that the letters of the two-loop NMHV octagon are included in the smallest fan we considered in table 1. However it could be that beyond two loops also the MHV amplitude will need recourse to the same set of algebraic letters. It could also be that beyond two loops the NMHV amplitude will require a bigger set of letters, say those arising in the largest fan considered in table 1. Moreover, the $\mathrm{N}^{2} \mathrm{MHV}$ amplitude requires algebraic letters (the fourmass box letters) at one loop already. These four algebraic letters are included in the set of 18 multiplicatively algebraic letters found in [30]. It would be very interesting to explore all these amplitudes at higher loop orders than are currently known explicitly to understand the general structure better. In a companion paper [48] we investigate different fans for finite Grassmannian cluster algebras, in particular the case of $\operatorname{Gr}(4,7)$ where we discuss the relation of MHV and NMHV amplitudes to different possible choices of tropical fan.

\section{Acknowledgments}

We are grateful to Nima Arkani-Hamed and Mark Spradlin for discussions about the topics described here. JD, JF and CK are supported by ERC grant 648630 IQFT. This project has received funding from the European Research Council (ERC) under the European Union's Horizon 2020 research and innovation programme (grant agreement No. 724638) 
Open Access. This article is distributed under the terms of the Creative Commons Attribution License (CC-BY 4.0), which permits any use, distribution and reproduction in any medium, provided the original author(s) and source are credited.

\section{References}

[1] S. Fomin and A. Zelevinsky, Cluster algebras I: Foundations, J. Am. Math. Soc. 15 (2002) 497 [math/0104151].

[2] S. Fomin and A. Zelevinsky, Cluster algebras II: Finite type classification, Invent. Math. 154 (2003) 63 [math/0208229].

[3] S. Fomin and A. Zelevinsky, Cluster algebras IV: Coefficients, Compos. Math. 143 (2007) 112 [math/0602259].

[4] N. Arkani-Hamed, J.L. Bourjaily, F. Cachazo, A.B. Goncharov, A. Postnikov and J. Trnka, Grassmannian Geometry of Scattering Amplitudes, Cambridge University Press (2016), [DOI] [arXiv: 1212.5605] [INSPIRE].

[5] J. Golden, A.B. Goncharov, M. Spradlin, C. Vergu and A. Volovich, Motivic Amplitudes and Cluster Coordinates, JHEP 01 (2014) 091 [arXiv: 1305.1617] [INSPIRE].

[6] J. Golden, M.F. Paulos, M. Spradlin and A. Volovich, Cluster Polylogarithms for Scattering Amplitudes, J. Phys. A 47 (2014) 474005 [arXiv:1401.6446] [INSPIRE].

[7] A.B. Goncharov, M. Spradlin, C. Vergu and A. Volovich, Classical Polylogarithms for Amplitudes and Wilson Loops, Phys. Rev. Lett. 105 (2010) 151605 [arXiv:1006.5703] [INSPIRE].

[8] L.J. Dixon, J.M. Drummond and J.M. Henn, Bootstrapping the three-loop hexagon, JHEP 11 (2011) 023 [arXiv: 1108.4461] [INSPIRE].

[9] L.J. Dixon, J.M. Drummond, M. von Hippel and J. Pennington, Hexagon functions and the three-loop remainder function, JHEP 12 (2013) 049 [arXiv: 1308.2276] [INSPIRE].

[10] L.J. Dixon, J.M. Drummond, C. Duhr and J. Pennington, The four-loop remainder function and multi-Regge behavior at NNLLA in planar $N=4$ super-Yang-Mills theory, JHEP 06 (2014) 116 [arXiv:1402.3300] [INSPIRE].

[11] L.J. Dixon and M. von Hippel, Bootstrapping an NMHV amplitude through three loops, JHEP 10 (2014) 065 [arXiv: 1408.1505] [INSPIRE].

[12] L.J. Dixon, M. von Hippel and A.J. McLeod, The four-loop six-gluon NMHV ratio function, JHEP 01 (2016) 053 [arXiv: 1509.08127] [INSPIRE].

[13] S. Caron-Huot, L.J. Dixon, A. McLeod and M. von Hippel, Bootstrapping a Five-Loop Amplitude Using Steinmann Relations, Phys. Rev. Lett. 117 (2016) 241601 [arXiv: 1609.00669] [INSPIRE].

[14] S. Caron-Huot, L.J. Dixon, F. Dulat, M. von Hippel, A.J. McLeod and G. Papathanasiou, Six-Gluon amplitudes in planar $\mathcal{N}=4$ super-Yang-Mills theory at six and seven loops, JHEP 08 (2019) 016 [arXiv: 1903.10890] [INSPIRE].

[15] J.M. Drummond, G. Papathanasiou and M. Spradlin, A Symbol of Uniqueness: The Cluster Bootstrap for the 3-Loop MHV Heptagon, JHEP 03 (2015) 072 [arXiv: 1412.3763] [INSPIRE]. 
[16] L.J. Dixon, J. Drummond, T. Harrington, A.J. McLeod, G. Papathanasiou and M. Spradlin, Heptagons from the Steinmann Cluster Bootstrap, JHEP 02 (2017) 137 [arXiv:1612.08976] [INSPIRE].

[17] J. Drummond, J. Foster, O. Gürdoğan and G. Papathanasiou, Cluster adjacency and the four-loop NMHV heptagon, JHEP 03 (2019) 087 [arXiv: 1812.04640] [INSPIRE].

[18] J. Drummond, J. Foster and O. Gürdoğan, Cluster Adjacency Properties of Scattering Amplitudes in $N=4$ Supersymmetric Yang-Mills Theory, Phys. Rev. Lett. 120 (2018) 161601 [arXiv: 1710.10953] [INSPIRE].

[19] J. Drummond, J. Foster and O. Gürdoğan, Cluster adjacency beyond MHV, JHEP 03 (2019) 086 [arXiv: 1810.08149 ] [INSPIRE].

[20] S. Caron-Huot, L.J. Dixon, F. Dulat, M. Von Hippel, A.J. McLeod and G. Papathanasiou, The Cosmic Galois Group and Extended Steinmann Relations for Planar $\mathcal{N}=4 S Y M$ Amplitudes, JHEP 09 (2019) 061 [arXiv: 1906.07116] [INSPIRE].

[21] S. Caron-Huot, Superconformal symmetry and two-loop amplitudes in planar $N=4$ super Yang-Mills, JHEP 12 (2011) 066 [arXiv:1105.5606] [INSPIRE].

[22] A. Hodges, Eliminating spurious poles from gauge-theoretic amplitudes, JHEP 05 (2013) 135 [arXiv:0905.1473] [INSPIRE].

[23] I. Prlina, M. Spradlin, J. Stankowicz, S. Stanojevic and A. Volovich, All-Helicity Symbol Alphabets from Unwound Amplituhedra, JHEP 05 (2018) 159 [arXiv:1711.11507] [INSPIRE].

[24] I. Prlina, M. Spradlin, J. Stankowicz and S. Stanojevic, Boundaries of Amplituhedra and NMHV Symbol Alphabets at Two Loops, JHEP 04 (2018) 049 [arXiv: 1712.08049] [INSPIRE].

[25] T. Dennen, I. Prlina, M. Spradlin, S. Stanojevic and A. Volovich, Landau Singularities from the Amplituhedron, JHEP 06 (2017) 152 [arXiv:1612.02708] [INSPIRE].

[26] N. Arkani-Hamed and J. Trnka, The Amplituhedron, JHEP 10 (2014) 030 [arXiv:1312.2007] [INSPIRE].

[27] N. Arkani-Hamed, H. Thomas and J. Trnka, Unwinding the Amplituhedron in Binary, JHEP 01 (2018) 016 [arXiv: 1704.05069] [INSPIRE].

[28] J.L. Bourjaily, A.J. McLeod, M. von Hippel and M. Wilhelm, Rationalizing Loop Integration, JHEP 08 (2018) 184 [arXiv: 1805.10281] [INSPIRE].

[29] J.L. Bourjaily, A.J. McLeod, C. Vergu, M. Volk, M. Von Hippel and M. Wilhelm, Rooting Out Letters: Octagonal Symbol Alphabets and Algebraic Number Theory, JHEP 02 (2020) 025 [arXiv: 1910.14224] [INSPIRE].

[30] S. He, Z. Li and C. Zhang, Two-loop Octagons, Algebraic Letters and $\bar{Q}$ Equations, Phys. Rev. D 101 (2020) 061701 [arXiv: 1911.01290] [INSPIRE].

[31] S. Caron-Huot and S. He, Jumpstarting the All-Loop S-matrix of Planar N $=4$ Super Yang-Mills, JHEP 07 (2012) 174 [arXiv:1112.1060] [INSPIRE].

[32] M. Bullimore and D. Skinner, Descent Equations for Superamplitudes, arXiv:1112.1056 [INSPIRE].

[33] L.F. Alday and J.M. Maldacena, Gluon scattering amplitudes at strong coupling, JHEP 06 (2007) 064 [arXiv:0705.0303] [INSPIRE]. 
[34] J.M. Drummond, G.P. Korchemsky and E. Sokatchev, Conformal properties of four-gluon planar amplitudes and Wilson loops, Nucl. Phys. B 795 (2008) 385 [arXiv:0707.0243] [INSPIRE].

[35] A. Brandhuber, P. Heslop and G. Travaglini, $M H V$ amplitudes in $N=4$ super Yang-Mills and Wilson loops, Nucl. Phys. B $\mathbf{7 9 4}$ (2008) 231 [arXiv:0707.1153] [InSPIRE].

[36] L.J. Mason and D. Skinner, The Complete Planar S-matrix of $N=4$ SYM as a Wilson Loop in Twistor Space, JHEP 12 (2010) 018 [arXiv:1009.2225] [INSPIRE].

[37] S. Caron-Huot, Notes on the scattering amplitude/Wilson loop duality, JHEP 07 (2011) 058 [arXiv: 1010.1167] [InSPIRE].

[38] J. Drummond, J. Foster, O. Gürdogan and C. Kalousios, Tropical Grassmannians, cluster algebras and scattering amplitudes, JHEP 04 (2020) 146 [arXiv:1907.01053] [INSPIRE].

[39] F. Cachazo, N. Early, A. Guevara and S. Mizera, Scattering Equations: From Projective Spaces to Tropical Grassmannians, JHEP 06 (2019) 039 [arXiv:1903.08904] [INSPIRE].

[40] D. Speyer and L.K. Williams, The tropical totally positive Grassmannian, math/0312297.

[41] N. Arkani-Hamed, T. Lam and M. Spradlin, Non-perturbative geometries for planar $\mathcal{N}=4$ SYM amplitudes, JHEP 03 (2021) 065 [arXiv: 1912.08222] [INSPIRE].

[42] N. Henke and G. Papathanasiou, How tropical are seven- and eight-particle amplitudes?, JHEP 08 (2020) 005 [arXiv: 1912.08254] [InSPIRE].

[43] A. Felikson, M. Shapiro and P. Tumarkin, Skew-symmetric cluster algebras of finite mutation type, J. Eur. Math. Soc. (2012) 1135.

[44] J.S. Scott, Grassmannians and cluster algebras, Proc. Lond. Math. Soc. 92 (2006) 345.

[45] N. Reading, A combinatorial approach to scattering diagrams, arXiv:1806.05094.

[46] J.M. Drummond, J. Henn, G.P. Korchemsky and E. Sokatchev, Dual superconformal symmetry of scattering amplitudes in $N=4$ super-Yang-Mills theory, Nucl. Phys. B $\mathbf{8 2 8}$ (2010) 317 [arXiv:0807.1095] [INSPIRE].

[47] D. Speyer and B. Sturmfels, The tropical Grassmannian, Adv. Geom. 4 (2004) 389.

[48] J. Drummond, J. Foster, O. Gürdoğan and C. Kalousios, Tropical fans, scattering equations and amplitudes, arXiv:2002.04624 [INSPIRE]. 